
\title{
$\checkmark$ Research Square \\ Proteoglycan-depleted regions of anular-injury promote nerve ingrowth in a rabbit disc degeneration model
}

\section{Long Xin}

Tongde Hospital Of Zhejiang Province https://orcid.org/0000-0001-9991-5240

\section{Weixing Xu}

Tongde Hospital Of Zhejiang Province

\section{Jian Wang}

Tongde Hospital Of Zhejiang Province

\section{Fang Yu}

Tongde Hospital Of Zhejiang Province

\section{Shunwu Fan}

Zhejiang University School of Medicine Sir Run Run Shaw Hospital

\section{Wei Wang}

Tianjin University of Science and Technology

Jian Liu

Tongde Hospital Of Zhejiang Province

yang yang ( $\square$ yangyanghz20@outlook.com )

\section{Research}

Keywords: PLGA/fibrin scaffold, Chondroitinase ABC, proteoglycan, nerve ingrowth disc degeneration

Posted Date: March 27th, 2020

DOl: https://doi.org/10.21203/rs.3.rs-19538/v1

License: (c) (i) This work is licensed under a Creative Commons Attribution 4.0 International License. Read Full License 


\section{Abstract}

\section{Background}

To assess the effects of proteoglycan-depleted regions of disrupted annulus on nerves ingrowth into the injury site in Vivo. Our study was designed to provide a test group of polymeric carriers loaded with chABC, using to enzymatically digest CSPGs in a controlled manner at the site of injury. And 2 control groups, one loaded with PBS buffer alone or the other created annular defect.

\section{Methods}

New Zealand white rabbits $(n=18)$ received annular injuries at $L 3 / 4, L 4 / 5$, and $L 5 / 6$. The experimental discs were randomly assigned to four groups: a) annular defect was created $(1.8 \mathrm{~mm}$ diameter; $4 \mathrm{~mm}$ depth); b) annular defect implanted PLGA/fibrin/PBS plug; c) annular defect implanted with a PLGA/fibrin/chABC plug; d) uninjured L2/3 disc (control). Disc degeneration was evaluated by radiography, MRI, histology, and analysis of proteoglycan content. And immunohistochemical detection of nerve fibers and chondroitin sulfate was respectively performed with PGP9.5 and CS-56.

Results

The radiographic, MRI, histological and biochemical changes demonstrated that the injured discs produced progressive and reliable disc degeneration. In the defect discs, lamellated appearance of AF has been replaced by extensive fibrocartilaginous-like tissue formed outside the injured sites. In contrast to be distributed along small fissures within newly formed tissue accompanied with small blood vessels appeared in the outer part of disrupted area in the PLGA/fibrin/PBS discs. Following chABC effectively enzymic deglycosylation, the residual scaffolds were surrounded by newly formed tissues, where more sprouting nerve fibers grew further into the depleted annulus regions in the PLGA/fibrin/chABC discs than control discs and those receiving PLGA/fibrin/PBS. In addition, innervations scores in the PLGA/fibrin/chABC discs showed significantly higher than those of PLGA/fibrin/PBS discs and defected discs.

\section{Conclusions}

ChABC-based PLGA/fibrin gel achieved the bio-integration with native annulus tissue and providing a local source for sustained release of active chABC. Disc-derived proteoglycan inhibited nerves and blood vessels ingrowth were evidently abrogated by chABC enzymic deglycosylation in an annular-injured rabbit disc degeneration model. The inhibitory effects of Neural and vascular ingrowth are inversely associated with the proteoglycan content. CS secreted from discs are potential candidates that could be useful to reduce neurite growth associated with discogenic pain.

\section{Background}


Low back pain is an extremely common socioeconomic problems in orthopaedics, although the pathophysiology of this condition remains uncertain, degeneration of the intervertebral disc(IVD) is believed to be a major cause of low back pain[1-3]. The degenerative change ultimately results in increased matrix degradation, pro-inflammatory cytokine expression and inferior mechanical properties [4-6]. More recently, progressive neurovascular growth in the annular fissures and sensitization of nerve fibers has been observed in the degenerated IVD[7, 8]. Ingrowth of nociceptive nerve fibers deep within disrupted intervertebral discs are believed to be one of the potential sources of discogenic pain in clinical cases [9-12].

It has been shown that aggrecan, a proteoglycan found in the disc, may act as a barrier to inhibit nerve fiber growth in vivo/vitro [13-16]. However, the precise inhibitory mechanism remains poorly understood. In previous work we showed that a minimally invasive annulotomy-induced rabbit model was well established, an orientated growth-permissive nerves growing into annulus defects during the degenerative process. Also, loss of proteoglycan at the injury site involved in IVD degeneration have been observed to facilitate the infiltration of blood vessels and nerve fibers into disrupted disc tissue[17, 18].

Chondroitinase $A B C$ (chABC) is a well-studied bacteria-derived enzyme for being able to specifically depolymerize the glycosaminoglycan (GAG) side chains for the induction of mild disc degeneration and favor axonal regeneration by reduction of proteoglycan in the injury site [19-24]. According to our previous works, PLGA/fibrin constructs as delivery system have good performance on biocompatibility, safety, and biodegradability $[18,25]$, also in promoting in tissue repair without compromising the function of incorporating proteins [26-30]. Therefore, as a potential platform for the carrier of chABC, it should be a simple and desirable alternative to achieve local delivery in a controlled manner as to cleavage of GAGs via digestion overcome CSPG-mediated inhibition at the injured site of the degenerated IVD.

In the present study, we hypothesized that proteoglycan-depleted regions of disrupted annulus could facilitate nerves and blood vessels ingrowth into the deeper parts of degenerated disc in a rabbit animal model. Here, fibrin-based polymeric carriers loaded with chABC for its sustained local delivery in vivo were implanted into injured annular site, to evaluate the effects of reduction of proteoglycan on nerves ingrowth during the IVD degeneration.

\section{Methods}

\section{Preparation of PLGA-coated fibrin gel/ChABC constructs}

The PLGA sponges were fabricated by a porogen leaching method as reported previously[31]. The PLGA/fibrin/chABC constructs were prepared as follows. The fibrinogen was isolated from fresh human plasma and final concentration used in all the experiments as previously established protocols $[25,32]$. ChABC (2U, Sigma, St. Louis, MO) was dissolved in buffer with a final concentration of $0.10 \mathrm{U} / \mathrm{ml}$ according to the product instructions and previous reported[19, 33]. Thereafter, the fibrinogen $(20 \mathrm{mg} / \mathrm{mL})$ and $\operatorname{ch} A B C(0.10 \mathrm{U} / \mathrm{ml})$ solutions were homogenized and sterilized by filtering through syringe filters. The 
PLGA plugs of $1.8 \mathrm{~mm}$ diameter and $4 \mathrm{~mm}$ length were immersed into the homogeneous fibrinogen/chABC solution $(1 \mathrm{~mL})$ under reduced pressure. The composite constructs were lyophilized and imaged using a SEM, and subsequently stored at $-20^{\circ} \mathrm{C}$ until further use.

\section{Evaluation Of chABC Release From PLGA/fibrin In Vitro}

To obtain the release profiles of each PLGA/fibrin gel loaded with chABC was suspended in $100 \mu \mathrm{L}$ of PBS solution $(\mathrm{pH}, 7.4)$ in polypropylene tubes placed in a shaker bath $\left(37^{\circ} \mathrm{C}\right)$ at $100 \mathrm{rpm} .10 \mu \mathrm{L}$ of supernatant was collected from each PLGA/fibrin sample at predetermined time intervals $(1,2,3,4,5,7,9$, 12 and 14 days) and individually stored at $-20^{\circ} \mathrm{C}$. According to previously reported protocol[34], the solution was tested for enzymatic activity by measuring the formation of unsaturated disaccharides by degrading the chondroitin sulfate over time (Sigma enzymatic assay-EC 4.2.2.4 per manufacturer instructions). Finally, the percentage of sample absorbance relative to the standard was calculated, the composite construct's accumulated release kinetic curve was obtained.

\section{Animal Surgery}

A total of 18 New Zealand rabbits (age $8.60 \pm 1.25$ months, weight $3.42 \pm 0.16 \mathrm{~kg}$ ) were supplied by the Laboratory Animal Center of Zhejiang province. All aspects of the study were approved by our Institutional Animal Care and Use Committee. The rabbits were randomly allocated to 1, 3, and 6-month survival groups ( $n=6$ in each group). The procedure of surgical implantation was used as described previously[17]. The consecutive levels of the rabbit IVD including $L 3 / 4, L 4 / 5$, and $L 5 / 6$ were exposed by an anterolateral retroperitoneal approach. Annular injuries were randomly allocated to 4 disc levels: (1) Annular defects group: annular defects (diameter $1.8 \mathrm{~mm} ; 4 \mathrm{~mm}$ depth) were created by a mini-trephine, as previously described[17, 18]; (2) PLGA/fibrin/PBS group: annular defects were filled with a PLGA/fibrin gel plug loaded phosphate buffered saline; (3) PLGA/fibrin/chABC group: annular defects were filled with a PLGA/fibrin gel plug loaded chABC; (4) Intact group: the L2/3 disc served as uninjured control. Finally, the wound was closed in layers. Following surgery, the rabbits were permitted free cage activity and food and water ad libitum.

\section{Radiographic Analyses And Magnetic Resonance Imaging (MRI)}

X-rays were obtained under general anesthesia (sodium pentobarbital, $30 \mathrm{mg} / \mathrm{kg}$ ) at 1, 3, and 6 months after surgery ( $n=6$ per time point). Comparation of disc height index ( $\mathrm{DHI}$ ) was calculated as a ratio of the injured disc height to the sum of the height of the two adjacent vertebral bodies, as previously described[35, 36]. The DHI\% therefore were expressed as DHI normalized to the baseline preoperative measurement (postoperative $\mathrm{DHI} /$ baseline $\mathrm{DHI} \times 100$ ). In vivo MRI of the lumbar spine examinations were performed using a 1.5-T MRI system (Signa, General Electric, Milwaukee, Wisconsin). Midsagittal 
T2-weighted images were obtained for analysis as previously described by the Pfirrmann's classification scores[37] based on changes of degree and area of signal intensity. All measurements were done using the picture archiving and communication system (PACS) routinely used in the local hospital.

\section{Tissue Harvesting}

At 1,3 and 6 months after surgery $(n=6)$, the rabbits were euthanized by intravenous sodium pentobarbital overdose for histology and immunohistology analysis. The experimental IVDs (L2/3, L3/4, $L 4 / 5$, and $L 5 / 6$ ) were removed from each lumbar spine under sterile conditions. The specimens were then dissected sagittally and divided into two symmetric parts. From one half of each disc, the nucleus pulposus (NP) was bluntly separated from the AF and then snap-frozen in liquid nitrogen, with subsequent storage at $-80^{\circ} \mathrm{C}$ in preparation for s-GAG analysis. The other half was used for histological analysis.

\section{Sulfated-glycosaminoglycan Content Measurement}

Samples of the NP were isolated from each level discs at 6 months after surgery $(n=6)$. The amount of proteoglycan content was quantified using the DMMB assay[38, 39]. Briefly, each lyophilized sample was digested with $125 \mu \mathrm{g} / \mathrm{mL}$ papain (Sangon Inc, ShangHai; PRC) in sterile PBS, $5 \mathrm{mM}$ EDTA, and $5 \mathrm{mM}$ cysteine $\mathrm{HCl}$ at $\mathrm{pH} 6.8$ and $60^{\circ} \mathrm{C}$ overnight. After complete digestion, $20 \mu \mathrm{L}$ papain digest were added to $200 \mu \mathrm{L}$ of DMMB reagent, with absorbance detected at $520 \mathrm{~nm}$. Total sGAG in the disc for each group was normalized according to the tested DNA amount, and then the SGAG/DNA ratio was measured and reported. The s-GAG content of the nucleus was measured and expressed as a percent of dry disc weight.

\section{Histology And Immunohistochemistry}

The specimens were fixed in $10 \%$ formalin, decalcified in ethylenediamine tetraacetic acid (EDTA), and processed for paraffin sectioning. Blocks of tissue were paraffin-embedded and sectioned at a thickness of $5 \mu \mathrm{m}$. Sections of IVD samples were stained with hematoxylin/eosin (HE) to observe degenerative changes, or with safranin-0 staining for the assessment of the proteoglycan content. Alternatively, the sections were subjected to immunohistology for the nerve marker Protein gene product 9.5 (PGP9.5). CS56 immunostaining was used to identify intact chondroitin sulfate (CS). All stained sections were analyzed under an optical microscope (Leica Microscope, Wetzlar, Germany). Briefly, the epitopes for PGP9.5 immunohistology were first heat-induced retrieved. The sections were then blocked with hydrogen peroxide and $25 \%$ normal bovine serum albumin (BSA)/tris buffered saline (TBS), and incubated overnight at $4{ }^{\circ} \mathrm{C}$ with the primary antibody to mouse monoclonal antibody against human protein gene product 9.5 (diluted 1:80, Abcam, Cambridge, GB). For CS56 staining, the sections were subjected to blocking of the endogenous peroxidase activity with $0.5 \%$ hydrogen peroxide for $1 \mathrm{~h}$ and then washed three times in TBS. Then, blocking with $25 \%$ normal BSA for $1 \mathrm{~h}$ (both at RT), and overnight incubation at 
$4{ }^{\circ} \mathrm{C}$ with a primary antibody to mouse anti CS56 (diluted 1:100, Sigma, St. Louis, MO). The sections were then washed again and incubated in biotinylated goat anti-mouse IgG antibody (1:200; Vector Laboratories) overnight. The sections were then processed with the avidin-biotin amplification method with conjugated peroxidase (Vectastain ABC Elite Kit; Vector) and visualized with diaminobenzidine (DAB; Sigma). Sections were then counterstained with hematoxylin and mounted with Aquatex for light microscopy. Control immunoglobulins consistently yielded negative results. The ingrowth of immunoreactive nerve fibers in the specimen was scored using a previously described grading scale [18, 40].

\section{Statistical analysis}

Results were expressed as means \pm standard error of the mean. Statistical analysis was performed using SPSS 22.0 software (SPSS Inc., Chicago, IL, USA). Significant differences in the radiograph measurements were analyzed by repeated-measurement analysis of variance (ANOVA) and Fisher's Least Significant Difference (LSD) test. The effect of time after surgery was analyzed with the Kruskal-Wallis test. Mann-Whitney $U$ tests were used to analyze the MRI score, innervation, and biochemical data. The level of significance was set at $\mathrm{P}<0.05$.

\section{Results}

\section{PLGA/fibrin/ChABC morphology and ChABC release kinetics}

The fabricated PLGA sponges filled with fibrin gel and chABC-coating of the PLGA/fibrin construct demonstrated by SEM, are shown in Fig. (1a) and (1b). SEM micrographs showed that interconnected micropores with a mean pore size of $350 \mathrm{um}$. The profiles of chABC release from PLGA/fibrin scaffold over 14 days are shown in Fig. 1(c). The curve of chABC from fibrin-based carrier showed a significant initial burst release during the first five days, and the chABC release continued steadily thereafter. Overall, the cumulative release rate of active chABC was found to be reached about $80 \%$ after 8 days.

\section{Radiographic And MRI Assessment}

Compared with the normal control group, disc height of the injured groups showed a slow but progressive decrease, sustaining for up to 6 months. At 6 months, narrowing disc space and bridging osteophyte were apparently observed in the two scaffold groups (Fig. 2a-b). The DHI\% of the two scaffold groups and the AF defect group were significantly lower than that of normal control groups at all postoperative periods $(p<0.05)$, whereas no significant difference was observed between PLGA/fibrin/PBS and PLGA/fibrin/chABC group $(p>0.05)$. Notably, DHI\% in the defect group demonstrated a significant decrease overtime by $1,3,6$ months after surgery $(p<0.01)$ (Fig. 3 ). 
Serial MRI scans of rabbit lumbar were showed that MRI appearance of the normal control discs remained relatively constant. Progressive decreases of signal intensity in NP area were apparent for each of the injured discs at all time points in Fig. 2c-d. The grade of MRI in the injured groups were progressively higher than that in the normal control groups at the postoperative time point $(p<0.01)$. Additionally, both the MRI grade of AF defect and PLGA/fibrin/chABC discs were significantly higher than that of the PLGA/fibrin/PBS or control discs after surgery $(p<0.05)$. However, no significant differences in the MRI grade was found between the PLGA/fibrin/chABC and the AF defect group at 1, 6 months after surgery $(p>0.05)$ (Fig. 4).

\section{Sulfated-glycosaminoglycan Content Of Nucleus}

The s-GAG content of three injured groups decreased significantly compared to control group at postoperative different time-point $(p<0.01)$. And the s-GAG content of PLGA/fibrin/chABC group showed a significant decrease when compared with that of the PLGA/fibrin/PBS and AF defect groups after surgery $(p<0.05)$. The decrease of sGAG/DNA ratio observed in the AF defect group was significantly more pronounced than in the PLGA/fibrin/PBS group at 1,6 months after surgery $(p<0.05)$ (Fig. 5).

\section{Histologic Assessment}

At 6 months, HE staining demonstrated that the uninjured, healthy discs (control group) were characteristically well-organized intact AF with its concentric lamellae. On safranin-O staining, uninjured discs showed minimal disruption of the proteoglycan matrix within the annulus (Fig. 6a, b). In the AF defect group, HE staining showed that loss of the lamellated appearance of AF, replacement of the annular defect by extensive fibrocartilaginous-like tissue formed outside the injured sites. Some blood vessels and small fissures at a limited depth were typically distributed in the outer scar tissue and AF, but not extending to the inner AF(Fig. $6 \mathrm{c})$. The safranin-0 staining indicated the presence of proteoglycan-rich content in the fibrocartilaginous tissue (Fig. 6d). In the PLGA/fibrin/PBS discs, HE staining showed small residues of PLGA scaffold with their naturally irregular form were enfolded by newly-formed tissue and well-integrated within the inner AF (Fig. 6e). In addition, safranin-0 staining showed open concave cavity increased severe loss of proteoglycan content (Fig. 6f). In contrast, advanced degeneration was observed in the PLGA/fibrin/chABC group, including NP fibrosis, disorganization of the AF and clusters of newly tissue extended further into the deeper inner AF along the fissures. Meanwhile, some blood vessels and small fissures were observed relatively common compared with that in PLGA/fibrin/PBS group (Fig. 6g). The proteoglycan content was markedly reduced in the injured region (Fig. 6h).

\section{CS-56 Immunolabeling For CS-GAG Digestion}

CS-56 immunolabeling in the lesion site indicated that chondroitin sulfate was degraded following treatment with chABC. In the control group, the outer lamellar AF was weakly stained with the CS-56 
antibody (Fig. 7a). In the defect discs, scar tissue was formed on the surface of the injury site. A fuzzy CS56-positive deposit was distributed in the scar tissue (Fig. 7b). In the PLGA/fibrin/PBS group, small residues of PLGA scaffold were surrounded by newly repair tissue penetrating deeper toward the NP. CS56-positive deposits were relatively negligible in the clusters of new regenerated tissue (Fig. 7c). In contrast, in the chABC/PLGA/fibrin-treated groups, CS-56 immunolabeling was high in the region of newly generated tissue at the lesion site but was intense reactivity overall (Fig. 7d).

\section{Innervation Of The Intervertebral Disc}

PGP9.5-positive nerves were seen sparsely in the lamellae and adjacent connective tissue of the outer AF (Fig. 8a). In the AF defect group, small nerves in the vicinity of the outer AF were distributed along the fissures, but barely seen to invade the deeper inner AF (Fig. 8b). In the PLGA/fibrin/PBS group, nerve fibers reactive for PGP9.5 were identified within the newly generated tissue of inner AF (Fig. 8c). In contrast, more sprouting PGP9.5-immunoreative fibers in the PLGA/fibrin/chABC group were localized predominantly in the vicinity of vascularized repair tissue extended further into the inner AF as well (Fig. 8d).

In terms of semi-quantitative innervation scores, three injured groups increased slightly over the follow-up period. Notably, Innervation grade of the PLGA/fibrin/chABC group was significantly higher than that in the AF defect or PLGA/fibrin/PBS group at 1,6 months after surgery $(p<0.05)$. The innervation score in the PLGA/fibrin/PBS group was significantly higher than that of AF defect group at 1 and 6 -month time point $(p<0.05)$, but no significant differences among the different time points $(p>0.05$; Fig. 9$)$.

\section{Discussion}

In our previous study $[17,18,41]$, an AF injury model was established by creating a defect, resulted in reproducible and degenerative MRI, radiograph, and histologic changes. Interestingly, degenerative changes occurred, characterized mainly by formation of the regenerated tissue and ingrowth of nerves and blood vessels into disrupted disc tissue. More recent studies have shown that aggrecan derived from both the AF and NP has inhibitory effect on nerve ingrowth into the IVD $[13,18,42]$. Thus, this experiment was conducted in an effort to evaluate the effects of degrading chondroitin sulphate (CS)-PG on neurite ingrowth by use of PLGA/fibrin gel incorporated with/without chABC was anchored in the annular defects during the degenerative process of the IVD. The results showed that fibrin-based PLGA loaded chABC exhibited to integrate well with native annulus tissue and provided a local source for sustained release of active chABC. Importantly, more nerve fibers were orientated conductively to grow into the deeper proteoglycan-depleted regions of disrupted annulus following chABC enzymic deglycosylation. To our best knowledge, there were few reports involving in vivo testing to assess the effects of proteoglycandepleted site in the annular wall on nerves ingrowth in a rabbit disc degeneration model.

It is well known that the action of chABC on proteoglycans was more specific degrades the chondroitin sulfate side chains of proteoglycans[24, 43$]$. Indeed, the use of proteolytic enzyme for the induction of 
mild disc degeneration has been successfully defined one of the modalities to achieve both in rabbits[44, $45]$ and in large animal sheep $[19,33]$. Furthermore, reduction of proteoglycan using fibrin-based delivery system achieving the enzymatic digestion depends on its dosage and activity[25, 46-48]. Based on the promising preliminary results, a dose of $0.1 \mathrm{U} / \mathrm{mL}$ chABC incorporated into PLGA/fibrin gel was determined in the present study. In line with previous studies[34, 49], the rate and duration of in vitro chABC release from the fibrin-based polymer was characterized by an initial burst $(70-80 \%)$ and presented in sustained manner up to 8 days. Thereafter, a small amount of chABC was still being released from the PLGA/fibrin gel, which achieved a delayed-release effect, as indicated in Fig. 1c. In addition, CS56-immunolabeling in the lesion site revealed that local distribution of chondroitin sulfate was degraded following treatment with chABC. Taken together, the results indicated that fibrin-based polymeric carriers in the injured site achieved the sustained, localized release of chABC, the activity of chABC in vivo was maintained after surgery, as shown by continuous significant decrease in the s-GAG content of PLGA/fibrin/chABC discs in comparison to 2 control discs. Conceivably, the present study rather undertakes a detailed analysis of the actual process of the glycosaminoglycan reduction and its activity, as it is very unlikely that the in vitro release kinetics would accurately reflect the more relevant in vivo release kinetics in the lesion site.

We observed PGP9.5-immunoreactive fibers in the defect group only in the superficial area of scar tissue, similar findings have been described in previous study[18]. However, increased nerve ingrowth into PGdepleted discs which sealed with PLGA/fibrin/chABC showed a significantly higher innervation score compared to PLGA/fibrin/PBS or empty defect discs. In addition, it indicated that advanced degeneration with small fissures and vascularization became more significant and penetrated deeper into the PLGA/fibrin/chABC discs in early postoperative period (see Fig. $6 \mathrm{~g}$ ). Eventually, degenerative procession appeared increasingly innervated in the chABC-treated discs following reduction of proteoglycan. Several factors potentially influence nerve ingrowth into the injured IVD: (i) more extensive proteoglycan reduction in the depleted annulus regions conducive to neural and vascular ingrowth following chABC enzymic deglycosylation; (ii) loss of structural integrity resulting in early leakage of NP with depletion of proteoglycan and increasing their attractiveness to ingrowing nerves; (iii) disruption of the tight collagen network that entraps the PGs and eventually more substantial newly tissue (e.g.,periannular innervated and vascularized granulation tissue) ingrowth along the local AF deficiency; (iv) ingrowth possibly inhibited by scar tissue due to less proteoglycan reduction than newly regenerated tissue in the AF injured track. A further limitation of using enzyme degradation in vivo model is that there could be still be local undegraded islands of CSPGs and be healed with the cells synthesizing and replenishing the lost GAGs. Thus, they do not completely mimic a natural 'degenerative' process. Additionally, further study is necessary to evaluate in the degenerated IVD for neural ingrowth in the application of the control enzyme (eg., hyaluronidase, Matrix Metalloproteinases) as a treatment option in a large animal model, with longer follow-up times and dose-related deglycosylation.

\section{Conclusion}


Our results indicate that chABC-based PLGA/fibrin gel showed promising results with regard to achieving the bio-integration with native annulus tissue and providing a local source for sustained release of active chABC. Disc proteoglycan inhibited nerves and blood vessels ingrowth were evidently abrogated by deglycosylation in an annular-injured rabbit disc degeneration model. Moreover, reduction of proteoglycan may play a direct role in nerves and blood vessels ingrowth into degenerated IVDs. Intact GAGs such as CS secreted from discs are potential candidates that could be useful to reduce neural and vascular ingrowth associated with discogenic pain in degenerated IVDs.

\section{List Of Abbreviations}

chABC chondroitinase $A B C$

PBS phosphate buffered saline

PLGA poly (lactic-co-glycolic acid)

PG proteoglycan

PGP9.5 protein gene product9.5

AF annulus fibrosus

IVD intervertebral disc

GAG glycosaminoglycan

MRI magnetic resonance imaging

DHI disc height index

NP nucleus pulposus

PACS picture archiving and communication system

DMMB 1,9-dimethylmethylene blue

EDTA ethylenediamine tetraacetic acid

HE hematoxylin/eosin

CS chondroitin sulfate

TBS tris buffered saline

ANOVA analysis of Variance 


\section{Declarations}

\section{Ethics approval and consent to participate}

All procedures performed in studies involving animals were approved by the institutional review board and animal care committee of Tongde Hospital. Protocols were conducted in accordance with the Guidance for the Care and Use of Laboratory Animals, as formulated by the Ministry of Science and Technology of the People's Republic of China, as well as the "Principles of laboratory animal care" (NIH publication No. 86-23, revised 1985) were followed.

\section{Consent for publication}

Individual person's data is not applicable in the study.

\section{Availability of data and materials}

All data are fully available without restriction.

\section{Competing interests}

The authors declare that they have no competing interests.

\section{Funding}

The authors gratefully acknowledge the financial support by Zhejiang Provincial Health commission of Foundation (2019KY050; No.2020362988) and by Zhejiang Provincial of Administration of Traditional Chinese Medicine of Foundation (2019ZA026; 2019ZB027 and 2019ZB029).

\section{Authors' contributions}

All authors were equally involved in conception and design of the study. LX, JL and WXX performed the surgical treatment and animal care. FY, JW and WW performed the acquisition, analysis and interpretation of the data. LX, YY and SWF finished the drafting of the manuscript and revised it critically for important intellectual content. All authors read and approved the final manuscript.

\section{Authors' information}

Long Xin is working as a spinal surgeon at department of Orthopedics, Tongde Hospital of Zhejiang Province, NO.234, Gucui Road, Hangzhou,310012, China. Yang yang is working as a professor at department of Orthopedics, Tongde Hospital of Zhejiang Province, and is the head of the Spinal Research Unit, Tongde Hospital.

\section{Acknowledgements}


The authors are grateful to Prof. Wei Wang for providing the PLGA, Youfa Shen for expert technical assistance, and to Dr. Ernesta Palombo-Kinne for critical review of the manuscript.

\section{References}

[1] N. Patrick, E. Emanski, M.A. Knaub, Acute and chronic low back pain, The Medical clinics of North America 98(4) (2014) 777-89, xii.

[2] D. Hoy, P. Brooks, F. Blyth, R. Buchbinder, The Epidemiology of low back pain, Best practice \& research. Clinical rheumatology 24(6) (2010) 769-81.

[3] S.P. Hughes, A.J. Freemont, D.W. Hukins, A.H. McGregor, S. Roberts, The pathogenesis of degeneration of the intervertebral disc and emerging therapies in the management of back pain, The Journal of bone and joint surgery. British volume 94(10) (2012) 1298-304.

[4] M.A. Adams, P.J. Roughley, What is intervertebral disc degeneration, and what causes it?, Spine 31(18) (2006) 2151-61.

[5] P. Colombier, J. Clouet, O. Hamel, L. Lescaudron, J. Guicheux, The lumbar intervertebral disc: from embryonic development to degeneration, Joint, bone, spine : revue du rhumatisme 81(2) (2014) 125-9.

[6] A.J. Freemont, The cellular pathobiology of the degenerate intervertebral disc and discogenic back pain, Rheumatology (Oxford, England) 48(1) (2009) 5-10.

[7] M. Stefanakis, M. Al-Abbasi, I. Harding, P. Pollintine, P. Dolan, J. Tarlton, M.A. Adams, Annulus fissures are mechanically and chemically conducive to the ingrowth of nerves and blood vessels, Spine 37(22) (2012) 1883-91.

[8] Y. Aoki, A. Nakajima, S. Ohtori, H. Takahashi, F. Watanabe, M. Sonobe, F. Terajima, M. Saito, K. Takahashi, T. Toyone, A. Watanabe, T. Nakajima, M. Takazawa, K. Nakagawa, Increase of nerve growth factor levels in the human herniated intervertebral disc: can annular rupture trigger discogenic back pain?, Arthritis research \& therapy 16(4) (2014) R159.

[9] A.J. Freemont, T.E. Peacock, P. Goupille, J.A. Hoyland, J. O'Brien, M.I. Jayson, Nerve ingrowth into diseased intervertebral disc in chronic back pain, Lancet (London, England) 350(9072) (1997) 178-81.

[10] S. Ohtori, G. Inoue, M. Miyagi, K. Takahashi, Pathomechanisms of discogenic low back pain in humans and animal models, The spine journal ,15(6) (2015) 1347-55.

[11] W.R.M. FRCA, Intervertebral disc as a source of pain, Continuing Education in Anaesthesia, Critical Care \& Pain Advance (2012).

[12] A.L. Binch, A.A. Cole, L.M. Breakwell, A.L. Michael, N. Chiverton, L.B. Creemers, A.K. Cross, C.L. Le Maitre, Nerves are more abundant than blood vessels in the degenerate human intervertebral disc, 
Arthritis research \& therapy 17 (2015) 370.

[13] W.E. Johnson, B. Caterson, S.M. Eisenstein, S. Roberts, Human intervertebral disc aggrecan inhibits endothelial cell adhesion and cell migration in vitro, Spine 30(10) (2005) 1139-47.

[14] W.E. Johnson, B. Caterson, S.M. Eisenstein, D.L. Hynds, D.M. Snow, S. Roberts, Human intervertebral disc aggrecan inhibits nerve growth in vitro, Arthritis and rheumatism 46(10) (2002) 2658-64.

[15] D. Purmessur, M.C. Cornejo, S.K. Cho, P.J. Roughley, R.J. Linhardt, A.C. Hecht, J.C. latridis, Intact glycosaminoglycans from intervertebral disc-derived notochordal cell-conditioned media inhibit neurite growth while maintaining neuronal cell viability, The spine journal , 15(5) (2015) 1060-9.

[16] J. Melrose, S. Roberts, S. Smith, J. Menage, P. Ghosh, Increased nerve and blood vessel ingrowth associated with proteoglycan depletion in an ovine anular lesion model of experimental disc degeneration, Spine 27(12) (2002) 1278-85.

[17] L. Xin, C. Zhang, F. Zhong, S. Fan, W. Wang, Z. Wang, Minimal invasive annulotomy for induction of disc degeneration and implantation of poly (lactic-co-glycolic acid) (PLGA) plugs for annular repair in a rabbit model, European journal of medical research 21(1) (2016) 7.

[18] L. Xin, W. Xu, L. Yu, S. Fan, W. Wang, F. Yu, Z. Wang, Effects of annulus defects and implantation of poly(lactic-co-glycolic acid) (PLGA)/fibrin gel scaffolds on nerves ingrowth in a rabbit model of annular injury disc degeneration, Journal of orthopaedic surgery and research 12(1) (2017) 73.

[19] S.E. Gullbrand, N.R. Malhotra, T.P. Schaer, Z. Zawacki, J.T. Martin, J.R. Bendigo, A.H. Milby, G.R. Dodge, E.J. Vresilovic, D.M. Elliott, R.L. Mauck, L.J. Smith, A large animal model that recapitulates the spectrum of human intervertebral disc degeneration, Osteoarthritis and cartilage 25(1) (2017) 146-156.

[20] Y. Shen, A.P. Tenney, S.A. Busch, K.P. Horn, F.X. Cuascut, K. Liu, Z. He, J. Silver, J.G. Flanagan, PTPsigma is a receptor for chondroitin sulfate proteoglycan, an inhibitor of neural regeneration, Science (New York, N.Y.) 326(5952) (2009) 592-6.

[21] S.C. Jefferson, N.J. Tester, D.R. Howland, Chondroitinase ABC promotes recovery of adaptive limb movements and enhances axonal growth caudal to a spinal hemisection, The Journal of neuroscience, 31(15) (2011) 5710-20.

[22] K. Zukor, Z. He, Regenerative medicine: drawing breath after spinal injury, Nature 475(7355) (2011) 177-8.

[23] J.C. Lee, H.J. Min, S. Lee, S.C. Seong, M.C. Lee, Effect of chondroitinase ABC on adhesion and behavior of synovial membrane-derived mesenchymal stem cells in rabbit partial-thickness chondral defects, Journal of orthopaedic research,31(8) (2013) 1293-301. 
[24] E. Muir, F. De Winter, J. Verhaagen, J. Fawcett, Recent advances in the therapeutic uses of chondroitinase ABC, Experimental neurology 321 (2019) 113032.

[25] W. Wang, B. Li, J. Yang, L. Xin, Y. Li, H. Yin, Y. Qi, Y. Jiang, H. Ouyang, C. Gao, The restoration of fullthickness cartilage defects with BMSCs and TGF-beta 1 loaded PLGA/fibrin gel constructs, Biomaterials 31(34) (2010) 8964-73.

[26] X.K. Li, S.X. Cai, B. Liu, Z.L. Xu, X.Z. Dai, K.W. Ma, S.Q. Lin, L. Yang, K.L. Sung, X.B. Fu, Characteristics of PLGA-gelatin complex as potential artificial nerve scaffold, Colloids and surfaces. B, Biointerfaces 57(2) (2007) 198-203.

[27] Y. Xiong, Y.S. Zeng, C.G. Zeng, B.L. Du, L.M. He, D.P. Quan, W. Zhang, J.M. Wang, J.L. Wu, Y. Li, J. Li, Synaptic transmission of neural stem cells seeded in 3-dimensional PLGA scaffolds, Biomaterials 30(22) (2009) 3711-22.

[28] D.Y. Lee, B.H. Choi, J.H. Park, S.J. Zhu, B.Y. Kim, J.Y. Huh, S.H. Lee, J.H. Jung, S.H. Kim, Nerve regeneration with the use of a poly(l-lactide-co-glycolic acid)-coated collagen tube filled with collagen gel, Journal of cranio-maxillo-facial surgery : official publication of the European Association for CranioMaxillo-Facial Surgery 34(1) (2006) 50-6.

[29] J. Han, P. Lazarovici, C. Pomerantz, X. Chen, Y. Wei, P.I. Lelkes, Co-electrospun blends of PLGA, gelatin, and elastin as potential nonthrombogenic scaffolds for vascular tissue engineering, Biomacromolecules 12(2) (2011) 399-408.

[30] Z. Pan, J. Ding, Poly(lactide-co-glycolide) porous scaffolds for tissue engineering and regenerative medicine, Interface focus 2(3) (2012) 366-77.

[31] G.Y. Zhou Q, Gao C. , Microstructure and mechanical properties of poly(l-lactide) scaffolds fabricated by gelatin particle leaching method, J Appl Polym Sci 98(3) (2005) 1373-9.

[32] A. Dresdale, E.A. Rose, V. Jeevanandam, K. Reemtsma, F.O. Bowman, J.R. Malm, Preparation of fibrin glue from single-donor fresh-frozen plasma, Surgery 97(6) (1985) 750-5.

[33] R.J. Hoogendoorn, P.I. Wuisman, T.H. Smit, V.E. Everts, M.N. Helder, Experimental intervertebral disc degeneration induced by chondroitinase ABC in the goat, Spine 32(17) (2007) 1816-25.

[34] A.J. Hyatt, D. Wang, J.C. Kwok, J.W. Fawcett, K.R. Martin, Controlled release of chondroitinase ABC from fibrin gel reduces the level of inhibitory glycosaminoglycan chains in lesioned spinal cord, Journal of controlled release,147(1) (2010) 24-9.

[35] K. Masuda, Y. Aota, C. Muehleman, Y. Imai, M. Okuma, E.J. Thonar, G.B. Andersson, H.S. An, A novel rabbit model of mild, reproducible disc degeneration by an anulus needle puncture: correlation between the degree of disc injury and radiological and histological appearances of disc degeneration, Spine 30(1) (2005) 5-14. 
[36] K. Masuda, Y. Imai, M. Okuma, C. Muehleman, K. Nakagawa, K. Akeda, E. Thonar, G. Andersson, H.S. An, Osteogenic protein-1 injection into a degenerated disc induces the restoration of disc height and structural changes in the rabbit anular puncture model, Spine 31(7) (2006) 742-54.

[37] C.W. Pfirrmann, A. Metzdorf, M. Zanetti, J. Hodler, N. Boos, Magnetic resonance classification of lumbar intervertebral disc degeneration, Spine 26(17) (2001) 1873-8.

[38] R.W. Farndale, D.J. Buttle, A.J. Barrett, Improved quantitation and discrimination of sulphated glycosaminoglycans by use of dimethylmethylene blue, Biochimica et biophysica acta 883(2) (1986) 173-7.

[39] L. Lao, H. Tan, Y. Wang, C. Gao, Chitosan modified poly(L-lactide) microspheres as cell microcarriers for cartilage tissue engineering, Colloids and surfaces. B, Biointerfaces 66(2) (2008) 218-25.

[40] Y. Aoki, K. Akeda, H. An, C. Muehleman, K. Takahashi, H. Moriya, K. Masuda, Nerve fiber ingrowth into scar tissue formed following nucleus pulposus extrusion in the rabbit anular-puncture disc degeneration model: effects of depth of puncture, Spine 31(21) (2006) E774-80.

[41] L. Xin, G.C. Han, F.D. Zhao, X. Zhao, G. Li, S.W. Fan, [In vivo study of innervation of degenerative intervertebral discs in rabbit anular-injury model], Journal of Zhejiang University. Medical sciences 38(5) (2009) 485-92.

[42] W.E. Johnson, S. Sivan, K.T. Wright, S.M. Eisenstein, A. Maroudas, S. Roberts, Human intervertebral disc cells promote nerve growth over substrata of human intervertebral disc aggrecan, Spine 31(11) (2006) 1187-93.

[43] H.I. Katsuhiko Ishibashi, Chemonucleolysis With Chondroitin Sulfate ABC Endolyase as a Novel Minimally Invasive Treatment for Patients With Lumbar Intervertebral Disc Herniation, J Spine Surg 5 (2019) S115-S121.

[44] J.P. Norcross, G.E. Lester, P. Weinhold, L.E. Dahners, An in vivo model of degenerative disc disease, Journal of orthopaedic research , 21(1) (2003) 183-8.

[45] Y. Imai, M. Okuma, H.S. An, K. Nakagawa, M. Yamada, C. Muehleman, E. Thonar, K. Masuda, Restoration of disc height loss by recombinant human osteogenic protein-1 injection into intervertebral discs undergoing degeneration induced by an intradiscal injection of chondroitinase ABC, Spine 32(11) (2007) 1197-205.

[46] L.J. Varden, D.T. Nguyen, A.J. Michalek, Slow depressurization following intradiscal injection leads to injectate leakage in a large animal model, JOR spine 2(3) (2019) e1061.

[47] P.C. Marion Fusellier, Longitudinal Comparison of Enzyme- And Laser-Treated Intervertebral Disc by MRI, X-Ray, and Histological Analyses Reveals Discrepancies in the Progression of Disc Degeneration: A Rabbit Study, BioMed research international (2016) 1-12. 
[48] G.D. O'Connell, R.J. Nims, J. Green, A.D. Cigan, G.A. Ateshian, C.T. Hung, Time and dose-dependent effects of chondroitinase ABC on growth of engineered cartilage, Eur Cell Mater 27 (2014) 312-320.

[49] J.-C. Lee, H.J. Min, S. Lee, S.C. Seong, M.C. Lee, Effect of chondroitinase ABC on adhesion and behavior of synovial membrane-derived mesenchymal stem cells in rabbit partial-thickness chondral defects, Journal of orthopaedic research,31(8) (2013) 1293-1301.

\section{Figures}
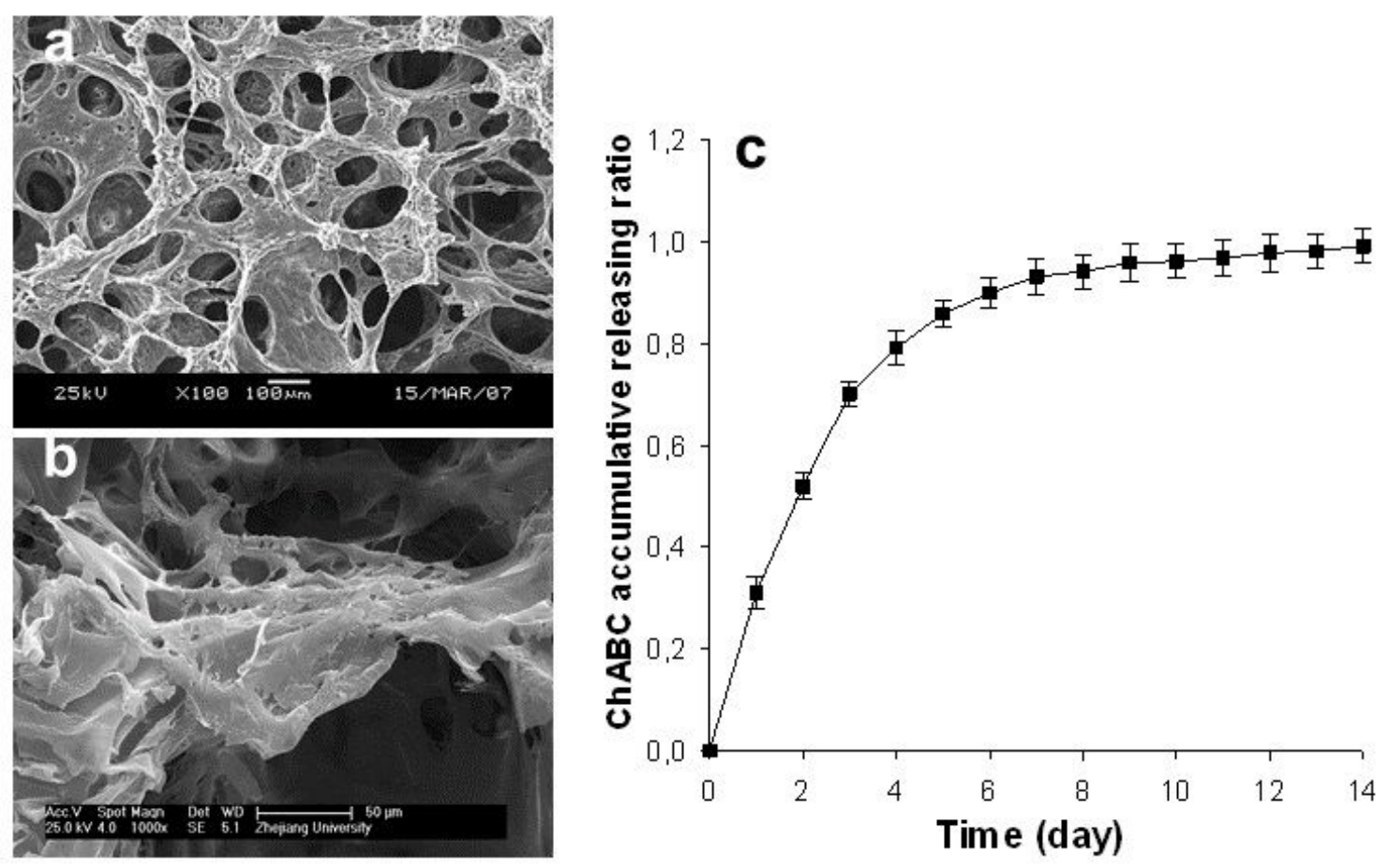

Figure 1

\section{Figure 1}

SEM micrographs of (a) PLGA/fibrin gel sponge; (b) the spongious composited construct filling fibrin gel and chABC with different magnification and in vitro cumulative release profile of chABC/PLGA/fibrin(c). 


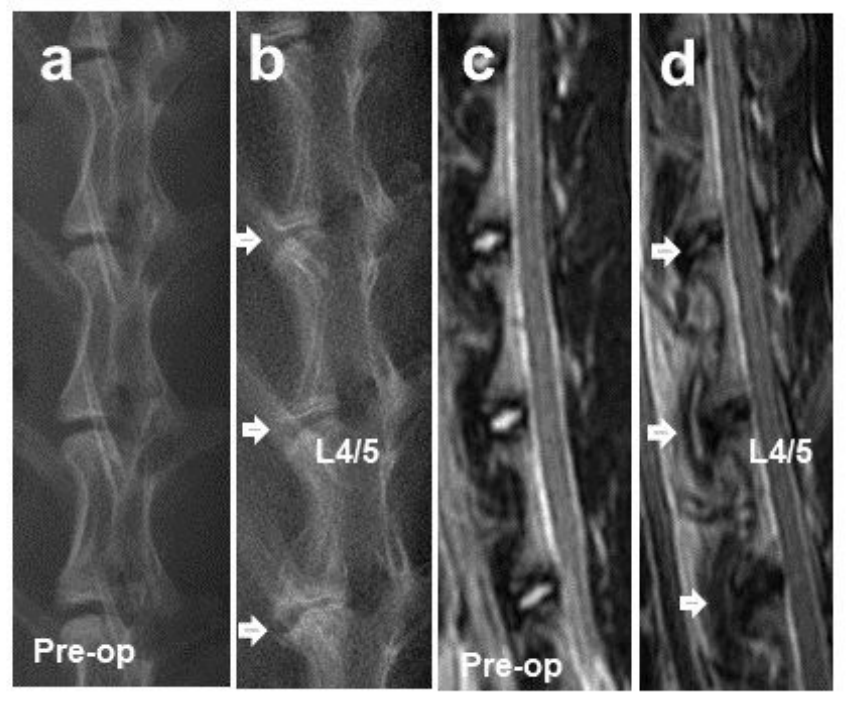

Figure 2

\section{Figure 2}

Representative lateral radiographs (a-b) and MRI(c-d) of rabbit lumbar spine taken 6 months after surgery. White arrows indicate the injured disc level. 


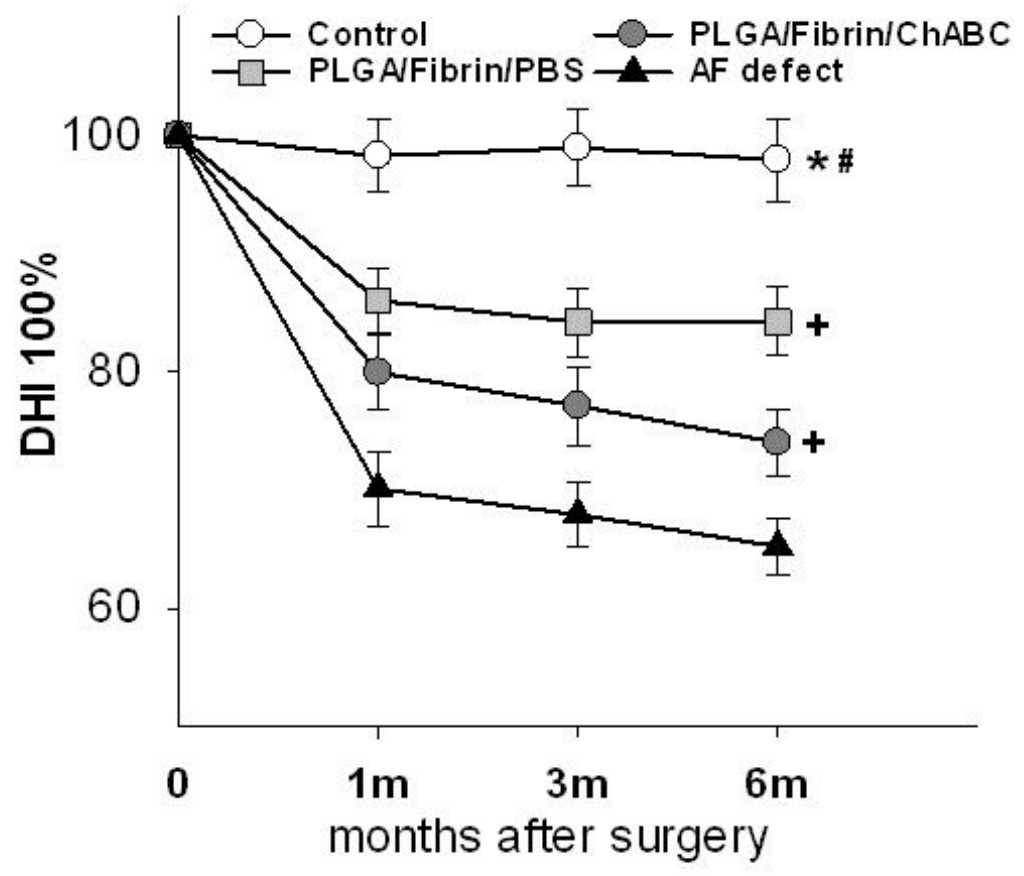

Figure 3

\section{Figure 3}

Changes in disc height index after surgery. There was a slow, progressive decrease in the disc height of operated discs over the follow-up period. The DHI in both two scaffold groups and the AF defect group were significantly lower than that of the control group after surgery $\left({ }^{*}, \# p<0.05\right.$, vs. the control group). $\mathrm{DHI} \%$ in the defect group demonstrated a significant decrease overtime by 1,3,6 months after surgery $(+p<0.01$, vs. the two scaffold groups). 


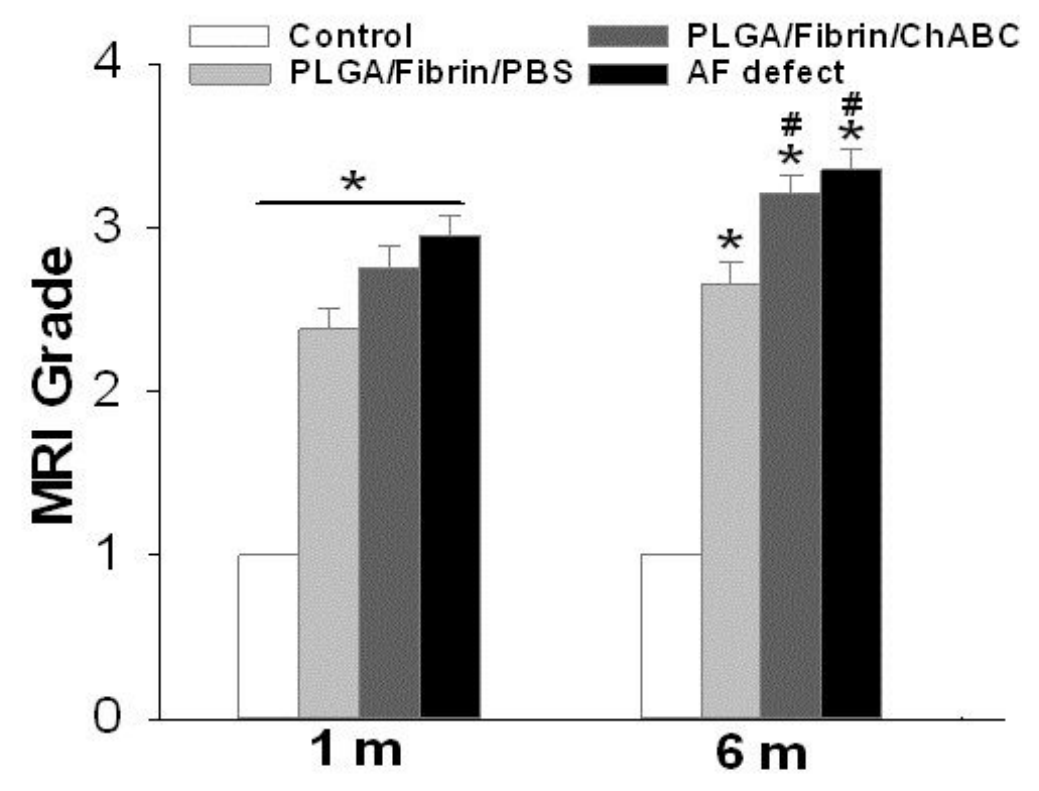

Figure 4

Figure 4

The grade of MRI in the injured groups were progressively higher than that in the normal control groups at the postoperative time point ( ${ }^{*} p<0.01$, vs. the control group). Both the MRI grade of AF defect and PLGA/fibrin/chABC discs were significantly higher than that of the PLGA/fibrin/PBS or control discs after surgery (\#p<0.05, vs. the control group or PLGA/fibrin/PBS group). 


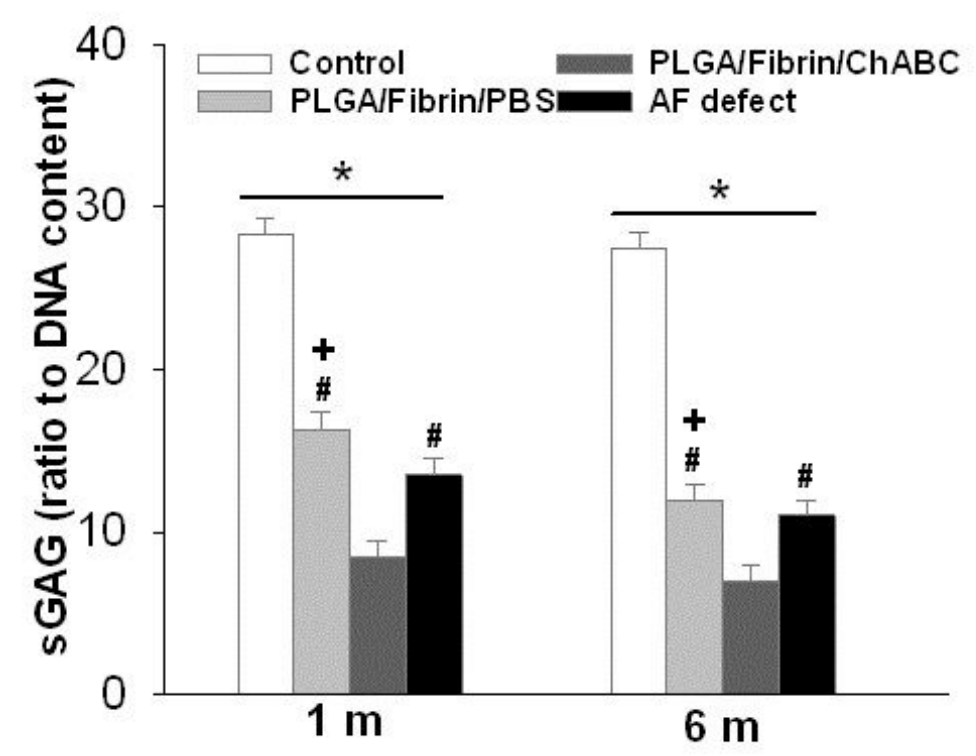

Figure 5

\section{Figure 5}

Changes in proteoglycan content of the NP at 6 months after surgery. The s-GAG content of three injured groups decreased significantly compared to control group at postoperative different time-point $\left({ }^{\star} p<0.01\right.$, vs. the control group). The PLGA/fibrin/chABC group showed a significant decrease of the sGAG/DNA ratio compared with the PLGA/fibrin/PBS or AF defect groups after surgery $(\# p<0.05$ vs. the PLGA/fibrin/PBS group or AF defect group). The decrease of sGAG/DNA ratio observed in the AF defect group was significantly more pronounced than in the PLGA/fibrin/PBS group at 1,6 months after surgery $(+p<0.05)$ 


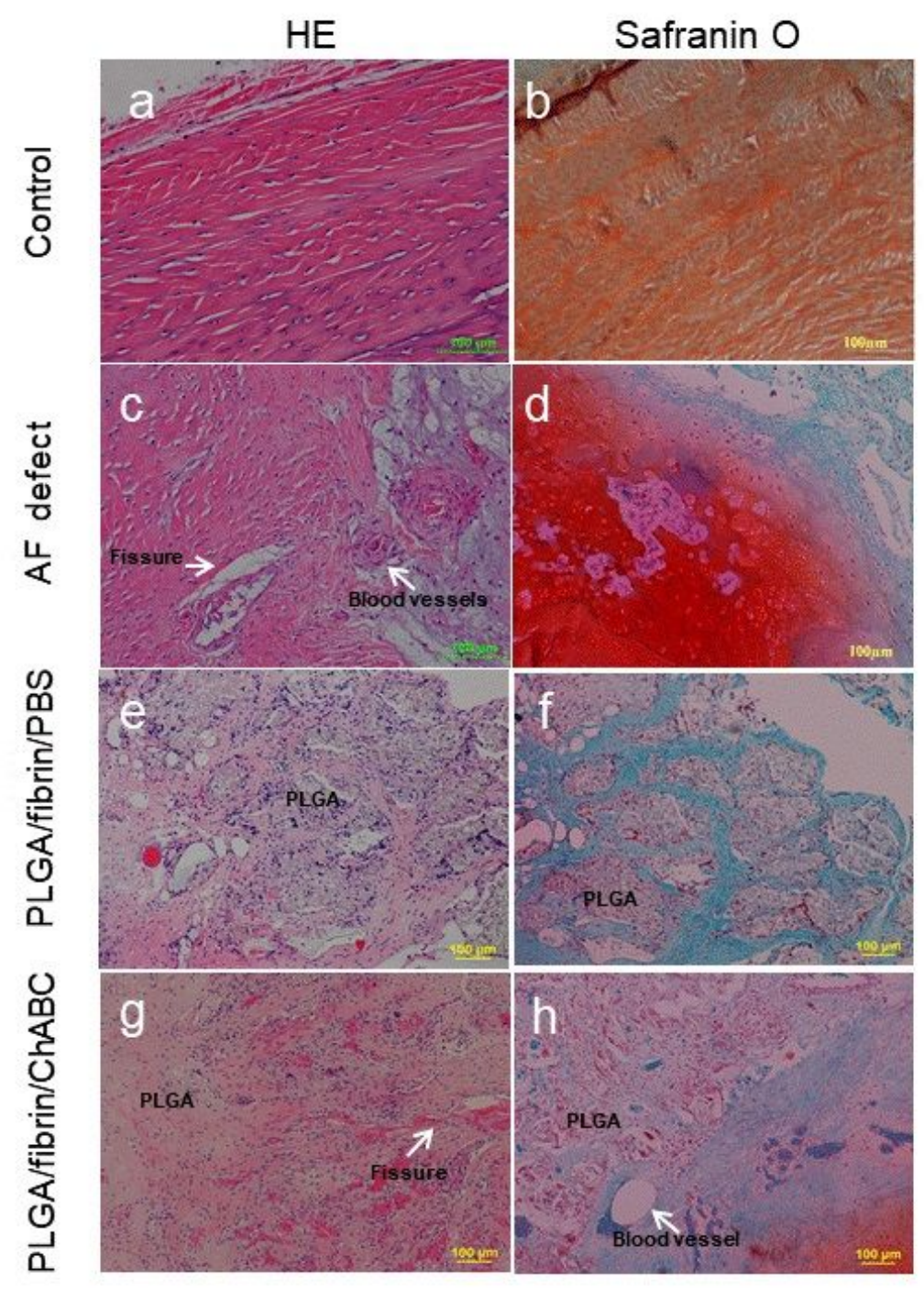

Figure 6

\section{Figure 6}

$\mathrm{HE}(\mathrm{a}, \mathrm{c}, \mathrm{e}, \mathrm{g})$ and safranin $\mathrm{O}(\mathrm{b}, \mathrm{d}, \mathrm{f}$ and $\mathrm{h})$ staining of the lesion site at 6 months after surgery. The uninjured AF displayed a multilamellar structure rich in proteoglycans, as shown by strong safranin 0 staining $(a, b)$. loss of the AF structural integrity, replacement by extensive fibrocartilaginous-like tissue in the injured sites. The safranin-O staining indicated the presence of proteoglycan-rich content in the fibrocartilaginous tissue $(c, d)$. Small irregular remnants of PLGA scaffold were well-integrated within the inner AF. Safranin-O staining showed open concave cavity increased severe loss of proteoglycan content $(e, f)$. advanced degeneration was observed in the PLGA/fibrin/chABC group, including NP fibrosis, disorganization of the $\mathrm{AF}$ and reparative tissue extended further into the deeper inner $\mathrm{AF}$ along the fissures (g). The proteoglycan content was markedly reduced in the injured region (h). White arrows indicate areas of some blood vessels and small fissures. 


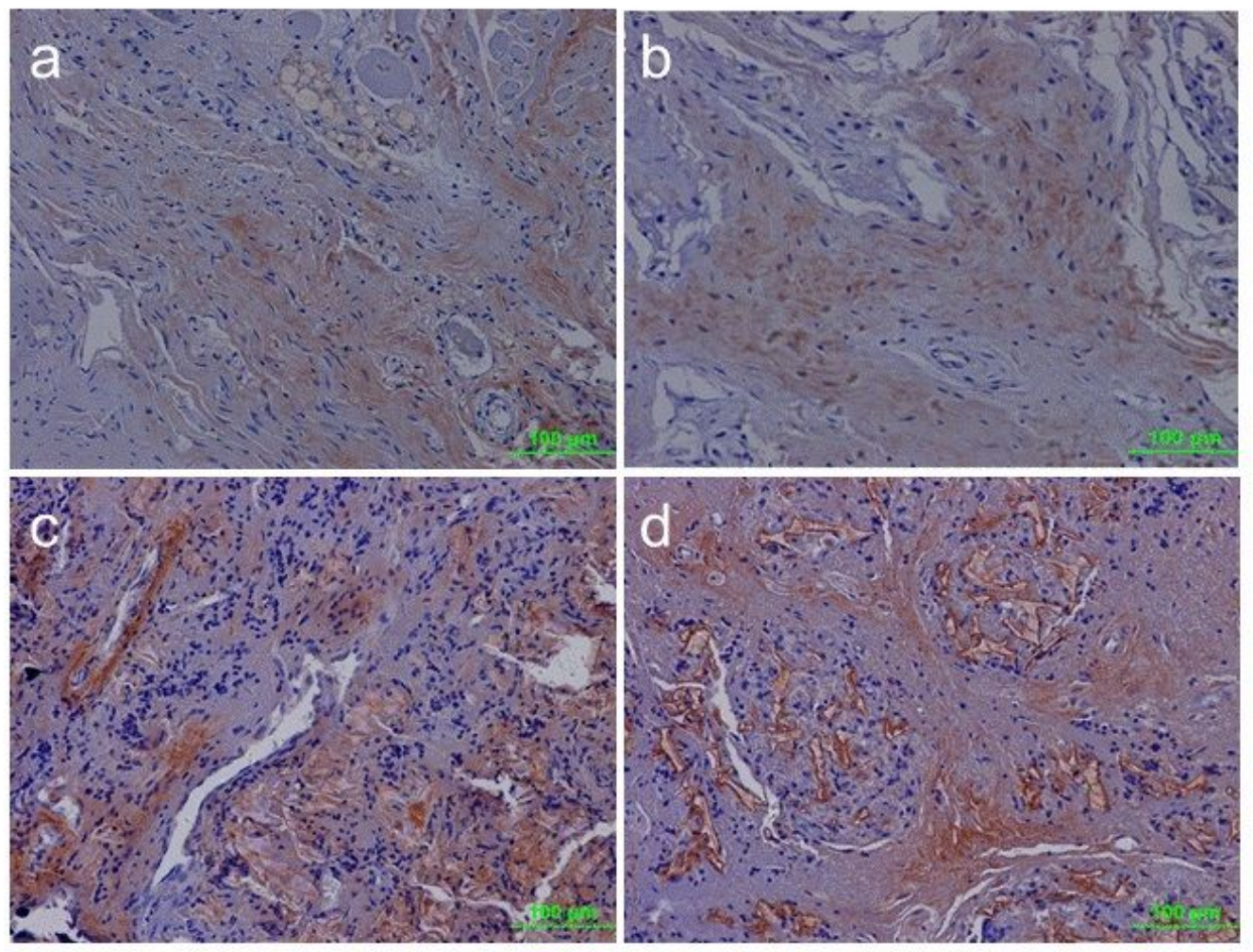

Figure 7

\section{Figure 7}

Immunohistochemical staining of CS56 in the lesion site. (a) the outer lamellar AF was weakly stained with the CS-56 antibody in the control group. (b) In the defect discs, scar tissue was formed on the surface of the injury site. A fuzzy CS56-positive deposit was distributed in the scar tissue. (c) In the PLGA/Fibrin/PBS discs, newly formed tissue penetrated deeper, toward the NP. CS56-positive deposits were relatively negligible in the reparative tissue. (d) In the PLGA/Fibrin/chABC discs, CS56 positivestaining showed intense reactivity at the site of injury following treatment with chABC. 


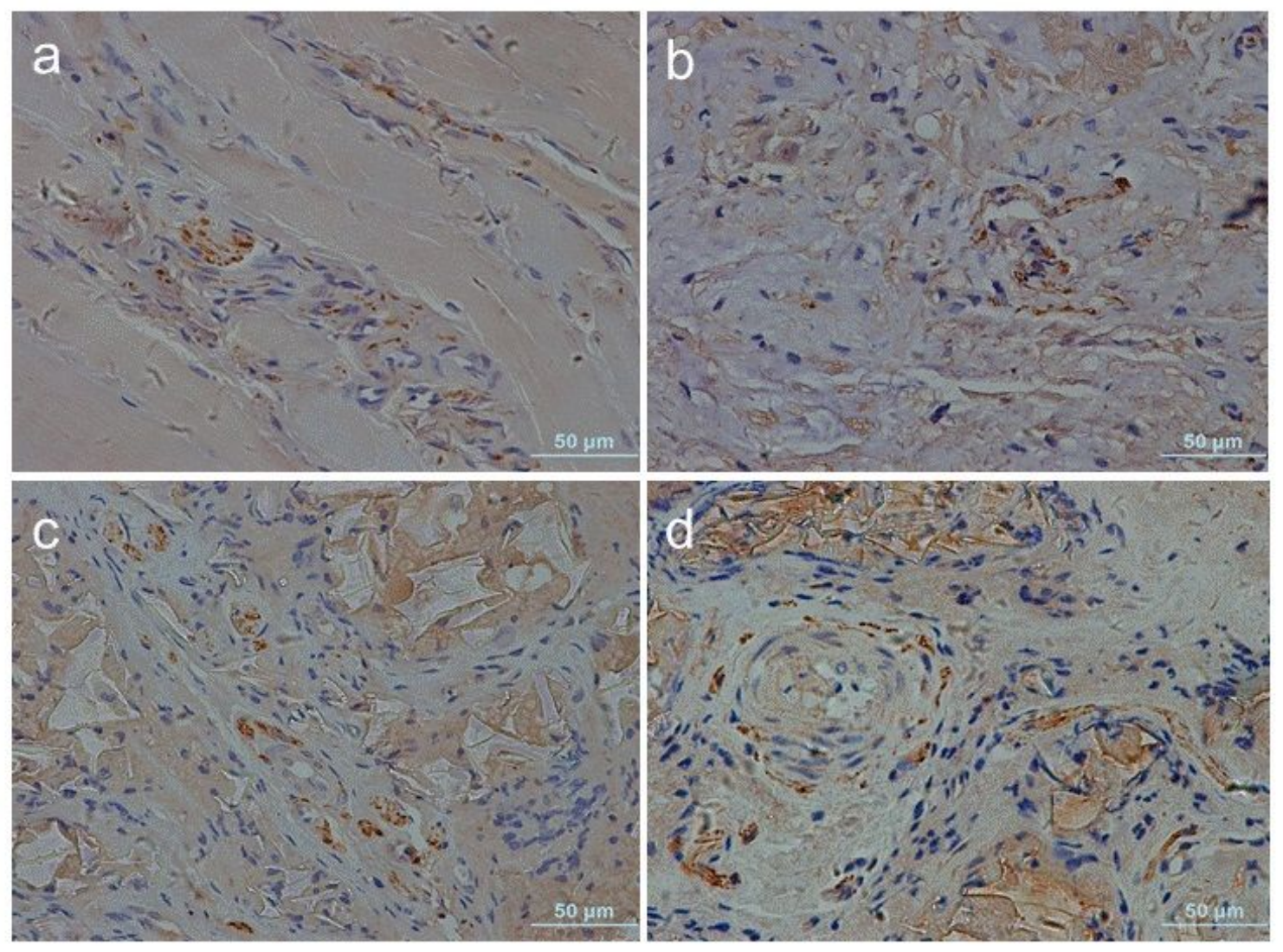

\section{Figure 8}

\section{Figure 8}

Immunohistochemical staining of PGP 9.5 at 6 months after surgery. The intact AF showed sparsely PGP 9.5- positive nerves distributed sparsely in the lamellae and adjacent connective tissue of the outer AF(a). Small nerves in the vicinity of the outer AF were distributed along the fissures, but barely seen to invade the deeper inner AF in the AF defect group (b). Nerve fibers reactive for PGP9.5 were present within the newly generated tissue of inner AF (c). In the PLGA/fibrin/chABC discs, more sprouting nerve fibers were predominantly identified in the vicinity of vascularized repair tissue extended further into the inner AF as well (d). 


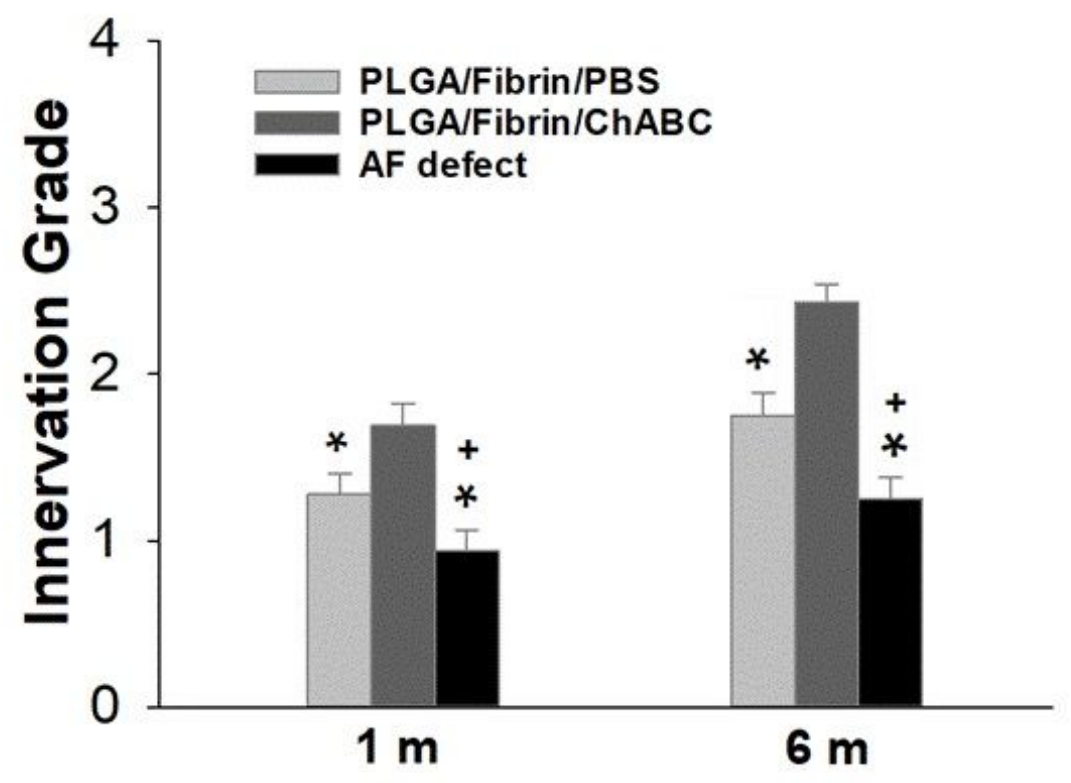

Figure 9

\section{Figure 9}

Changes in innervation scores at 6 months after surgery. The innervation score in the PLGA/fibrin/chABC group was significantly higher than that in the AF defect or PLGA/fibrin/PBS group at 1,6 months after surgery ( 1 and 6 months, ${ }^{*} p<0.01$ ). The innervation score of the PLGA/fibrin/PBS discs was significantly higher than that of AF defect discs at 1 and 6-month time point. ( $\mathrm{tp}<0.05 \mathrm{vs}$. the AF defect group).

\section{Supplementary Files}

This is a list of supplementary files associated with this preprint. Click to download.

- 0122020BMCARRIVEChecklist.pdf 